\title{
Study of Mucocutaneous Manifestations in Diabetes Mellitus
}

\author{
Dr. P. Satyanarayana Rao ${ }^{1}$. Dr.V. Srilakshmi ${ }^{2}$, Dr. Ch.Kishore Kumar ${ }^{3}$, \\ Dr.R. Vijaya Bhaskar ${ }^{4}$. \\ ${ }^{I}$ (Associate Professor, Depatment Of Pathology, Rangaraya Medical College, Kakinada, \\ Andhra Pradesh, India) \\ ${ }^{2}$ (Senior Resident, Depatment Of Pathology, Rangaraya Medical College, Kakinada, \\ Andhra Pradesh, India) \\ ${ }_{3}^{3}$ Assistant Professor, Depatment Of Pathology, Rangaraya Medical College, Kakinada, \\ Andhra Pradesh, India) \\ ${ }^{4}$ (Professor \& Head, Depatment Of Pathology, Rangaraya Medical College, Kakinada, \\ Andhra Pradesh, India)
}

\begin{abstract}
:
Introduction: Diabetes mellitus is the most common endocrine disorder with increasing incidence. $30 \%$ of diabetic persons have some type of mucocutaneous involvement from trivial to life threatening during the course of their chronic disease.

Aims Of The Study:To study the prevalence of mucocutaneous manifestations in Diabetes mellitus in our locality and to analyse the histopathological spectrum of these lesions.

Materails and Methods: It is a prospective study of diabetic patients with mucocutaneous lesions attended to Government General Hospital, Rangaraya Medical College, Kakinada over a period of two years from August 2013 - July 2015. By applying exclusion and inclusion criteria, we collected the material from 163 cases by FNAC, scrape cytology and punch biopsy depending on the type of lesion. Culture and sensitivity and gram staining were also done in necessary cases.

Results and analysis: After exclusion of 3 cases due to inadequate material obtained, total 160 cases results were analysed. Analysis show mucocutaneous lesions have slightly more predilection for Male gender (57\%) and age group between 51-60 yrs(41\%). Infections were the most common lesions in diabetics - constitutes $48 \%$, dermatophytes( $45 \%)$ and candida species (39\%) were the most common pathogens.
\end{abstract}

Discussion: Most of the parameters were correlated with other studies.

Conclusion: 1.Diabetics are susceptible to mucocutaneous lesions that are uncommon in non diabetics .

2. Whenever any patient present with multiple mucocutaneous manifestations, check for diabetes immediately.

Keywords: Diabetes Mellitus, Mucocutaneous lesions.

\section{Introduction}

Diabetes mellitus is the most common endocrine disorder with increasing incidence. International Diabetic Federation estimated that 60.1 million persons in India have Diabetes mellitus . It is further set to rise up to 100.6 million by the year $2030^{1}$.

It is characterised by hyperglycemia, disturbances of carbohydrate and lipid metabolism. Abnormal carbohydrate metabolism, atherosclerosis, microangiopathy, macroangiopathy, neuropathy and impaired host immune mechanisms all play a role in the pathogenesis of cutaneous complications ${ }^{1}$. Diabetes has a profound effect on both cell mediated and humoral immunity and also on the function of polymorphonuclear cells that predisposes these individuals to a variety of infections.

$30 \%$ of persons with diabetes have some type of mucocutaneous involvement during the course of their chronic disease. ${ }^{1}$ Mucocutaneous manifestations of diabetes mellitus are many and vary from trivial to life threatening. Sometimes mucocutaneous disorders may herald the onset of diabetes. Eg: Candidial balanoprosthitis, diabetic dermopathy, acrochordons, acanthosis nigricans, bullous diabeticorum .

\section{Material and methods}

It is a prospective study of patients having diabetes with skin and mucous membrane lesions attending Skin, Medical, ENT and Dental OPD'S and wards GGH, RMC, Kakinada over a period of two years from August 2013 - July 2015.

Total 176 cases were selected for the study. Out of 176 patients with mucocutaneous lesions, we collected the material from 163 cases only. The remaining 13 cases were patients with high blood glucose levels and patients who are non cooperative for the study. In 3 cases material obtained was inadequate, hence finally 160 cases were included in the present study. 
Detailed history was taken and clinical examination was done. Material was collected from the patients by FNAC, scrape cytology and punch biopsy depending on the type of lesion. Pus from diabetic ulcer was sent for culture and sensitivity wherever necessary. Gram staining was done for furunculosis(Fig. 6) and candidiasis(Fig. 4) cases.

Punch biopsy of size 4-5 mm was taken from the lesion, tissue was processed, paraffin blocks are prepared, and slides were stained with haematoxylin and eosin. Scrapings were collected from the lesion and $5 \% \mathrm{KOH}$ is added to them and observed for presence of fungal hyphae (Fig. 2).

\section{Results}

A total number of 160 cases were included in the study. Males show slight predominance over females (Table 1). Male patients were $91(57 \%)$ and female patients were $69(43 \%)$.

In age wise distribution of cases (Graph 1), 66(41\%) cases fall under the age group of 51-60 yrs was the highest age group involved, $44(28 \%)$ cases fall under 41-50 yrs age group was the next highest age group .

On classifying mucocutaneous lesions according to duration of diabetes (Table 3), majority of patients had 10 years duration constituting $64(40 \%)$ cases, minority of patients were newly diagnosed constituting 10 $(6 \%)$ cases.

On classifying mucocutaneous lesions based on number of lesions per patient (Table 2), Patients with single lesion were $114(72 \%)$, patients with two lesions were $38(23 \%)$ and patients with three or more lesions were $8(5 \%)$.

On classifying mucocutaneous lesions(Graph 2), Infections were the most common constituting 77(48\%) cases, followed by others like Psoriasis 62(39\%), neurological lesions 14 (8.7\%), Skin conditions with strong association $4(2.5 \%)$, Cutaneous reactions to drugs $2(1.2 \%)$, Disorder of collagen $1(0.6 \%)$.

Among infections(Graph 3) dermatophytes were the most common(Fig 1,2) and constitute 35 $(45.4 \%)$ cases, followed by candiasis 30(39\%)(Fig 3,4), Furunculosis 8(10.4\%)(Fig5,6), Mycobacterial infections 3(4\%), and Scabies $1(1.2 \%)$.

Neurological lesions include 14 cases of diabetic ulcers (Fig 7) with klebsiella followed by staphylococci (Fig 8) being the most commonly isolated organisms on culture and sensitivity. Disorders of collagen include one case of Granuloma anulare. Reactions to treatment include two cases of lichenoid drug eruption.

Other lesions constitute Sixty two cases. These include lesions like xerosis 20(12.5\%); Post inflammatory pigmentary alteration 9 (5.6\%); Polymorphic light eruption 4 (2.5\%); Psoriasis 3( $1.8 \%)$; basal cell carcinoma 3(1.8\%); Lichen simplex chronicus 3(1.8\%); Non specific dermatitis 3(1.8\%); Spongiotic dermatitis 2(1.2\%); Seborrheic keratosis 2(1.2\%); Seborrheic dermatitis 2(1.2\%); Acrochordons 2(1.2\%); Borderline tuberculoid Hansens 2(1.2\%); Lichen planus 1(0.6\%); Contact dermatitis 1(0.6\%); Fibroma $1(0.6 \%)$; chronic lichenified eczema $1(0.6 \%)$; Lupus vulgaris $1(0.6 \%)$; Squamous papilloma $1(0.6 \%)$; lichen amyloidosis $1(0.6 \%)$.

On classifying lesions according to duration of diabetes in patients with shorter duration infections were the most common followed by cutaneous reactions to drug treatment. Where as in patients with longer duration neurological lesions, others like psoriasis, acrochordon, post inflammatory pigmentary alterations and malignancies were the most common.

\section{Discussion}

In our study males show slight predominance over females (57\% and $43 \%$ ) and is correlating with the previous studies done by Abhisheck goel et.al., ${ }^{3}(54 \%$ and $46 \%)$ and Roshini vahora et.al., ${ }^{4}(51 \%$ and $49 \%)$ and it is not correlating with Mahajan et.al., ${ }^{1}$ study in which females show preponderance over males $(42 \%$ and $58 \%$ ). Most patients fall in the age group of 41- 60 years in the present study and is correlating with the previous studies done by Mahajan et. al., (41-50yrs) and Roshini vahora et.al., (>40 yrs). $28 \%$ of patients show more than one lesion in present study and is correlating with other studies done by Roshini et .al., ${ }^{4}(44 \%)$.

Infections were the most common lesions in the present study $(48 \%)$ and it is correlating with the previous studies done by N.T. Foss et.al., ${ }^{2}(82 \%)$ and Mahajan et.al., ${ }^{1}(55 \%)$. Roshini et.al., ${ }^{4}$ (39\%). Among infections fungal infections were the most common. Superficial mycoses (tineas) cause pruritus and its absence could suggest a compromised response in DM patients. It is due to impaired superficial innervations caused by diabetic neuropathy ${ }^{2}$. Usually Fungal agents colonizing in keratin layer of skin, does not cross a natural barrier created by corneal layer. For this they have to overcome fungistatic action of fatty acids produced by keratin cells. Deeper epidermal layers activate the immune response against infections. Both mechanisms are impaired in the skin of DM patients ${ }^{2}$. So there is increased incidence of fungal infections in diabetics. Diabetics with inadequate metabolic control had considerably more dermatophytoses and candidiasis than those with adequate metabolic control and is correlating with the study done by Gupta et.al., 
Xerosis is common (28\%) next to infections and is correlating with previous study done by Abhishek goel et al $(44 \%)^{3}$. Increased production and accumulation of free radicals or advanced glycosylation endproducts (AGE) in excess in the skin of DM patients is the cause ${ }^{2}$. In our study complications with anti-diabetic treatment were seen in $2 \%$ of patients and is correlating with the studies done by Bhat et al., ${ }^{11}(5 \%)$, Roshini vahora study (5\%), Mahajan et al. ${ }^{l}(4.69 \%)$. In present study $3(1.87 \%)$ patients had psoriasis. Sezai et al., reported psoriasis co existed with diabetes in $11.2 \%$ cases.

Acanthosis nigricans was seen in $2.5 \%$ patients in present study. Acanthosis nigricans was reported in $20 \%, 13 \%, 5 \%, 5.3 \%$ of cases in Bhargava et al. study, Roshini vahora study, Raghunatha et al., Study and Bhat et al., studies respectively. The first major break through association of acanthosis nigricans with insulin resistance came from a study by Kahn. Insulin at high concentrations may stimulate insulin-like growth factor receptors on keratinocytes, there by promoting epidermal cell proliferation. Every patient of Acanthosis nigricans should be investigated for diabetes and every patient of diabetes should be screened for acanthosis nigricans.

In contrary to other studies in which skin conditions like acanthosis nigricans, necrobiosis lipoidica, granuloma anulare were associated with diabetes, in the present study other lesions like psoriasis, postinflammatory pigmentary alterations, lichen planus, seborrheic dermatitis, spongiotic dermatitis, lichen amyloidosis cases were also observed.

On comparision most of the parameters were correlated with other studies (Table 4).

\section{Summary}

A prospective study with 160 cases of diabetics with mucocutaneous lesions was undertaken over a period of two years (August 2013 - July 2015) in the department of pathology, Rangaraya Medical college, Kakinada. At least $30 \%$ of diabetics have some type of mucocutaneous involvement during the course of their chronic disease. In present study males (57\%) show predominance over females (43\%). Most patients fall in the age group of 50-60 years (41\%). Most patients had 10 years duration of diabetes $(40 \%)$. Infections were the most common lesions (48\%). Among infection dermatophytes were the most common (45\%). In newly diagnosed diabetics infections were the most common where as in patients with longer duration neurological lesions were the most common. Next to infections, xerosis was the most common followed by others like psoriasis and post inflammatory pigmentary alterations. Prompt identification of these mucocutaneous lesions are important in the proper treatment and there by preventing further complications.

\section{Conclusion}

1.Diabetics are susceptible to mucocutaneous lesions that are uncommon in non diabetics. Many of such diseases require quick diagnosis and immediate treatment to avoid severe complications and to prevent fatality.

2. Whenever any patient presents with multiple mucocutaneous manifestations, check for diabetes immediately.

References

[1]. Mahajan S, Koranne RV, Sharma SK . Cutaneous manifestations of Diabetes melitus. Department of DermatoVenereology, Lady Harding Medical College and Thio Hospitals, New Delhi. Year : 2003 Volume : 69 Issue : 2 Page : 105-108.

[2]. N T Foss; D P Polon; M H Takada Skin lesions in diabetic patients. M C Foss- Freitas Departament of Clínical Médicine. Vol. 39 No:4, Aug. 2005.

[3]. Abhishek goyal, Sujeet Raina, Satinder S kaushal, Vikram Mahajan, Nand Lal Sharma Indian Journal of Dermatology. Patterns of cutaneous manifestations in diabetes mellitus. 2010 Jan-Mar; 39-41.John D. Bancroft, Alan Stevens, Theory and Practice of histological techniques; 2nd edition; 95- 122.

[4]. Roshini vahora,Sejal thakkar,Yogesh Marfatia.Department of Dermatology, Medical College and SSG Hospital, A longitudinal study in a tertiary care hospital in GujaratVadodara, Gujarat,.Skin, a mirror reflecting diabetes mellitus. Year : 2013 Volume : 17 Issue : 4 Page : 659-664.

[5]. Cutaneous manifestations of diabetes mellitus Journal of the American Academy of Dermatology Volume 30, Issue 4, Pages 519-531, April 1994.

[6]. Morgan AJ, Schwartz RA. Diabetic dermopathy: a subtle sign with grave implications.J Am Academy Dermato2008; 58: 447-451.

[7]. Romano G, Moretti G, Di Benedetto A, Giofre C, et al. Skin lesions in diabetes mellitus: prevalence and clinical correlations. Diabetes Research and Clinical Practice 1998;39:101-106. Sibbald RG. The skin and diabetes mellitus. International j ournal Dermatology 1984; 23: 567 .

[8]. Perez MI, Kohn SR. The cutaneous manifestations of diabetes mellitus.

[9]. JAAD 1986 JE 14: 612. Jelinek Dermatology 1994;30:519-529.

[10]. Romano G, Moretti G, Di Benedetto A, Giofre C, Di Cesare E, Russo G, et al. Skin lesions in diabetes mellitus: Prevalence and clinical correlations. Diabetes Res Clin Pract. 1998;39:101-6.

[11]. Bhat YJ, Gupta V, Kudyar RP. Cutaneous manifestations of diabetes mellitus. Int J Diab Dev Ctries.2006;26:152-5. 

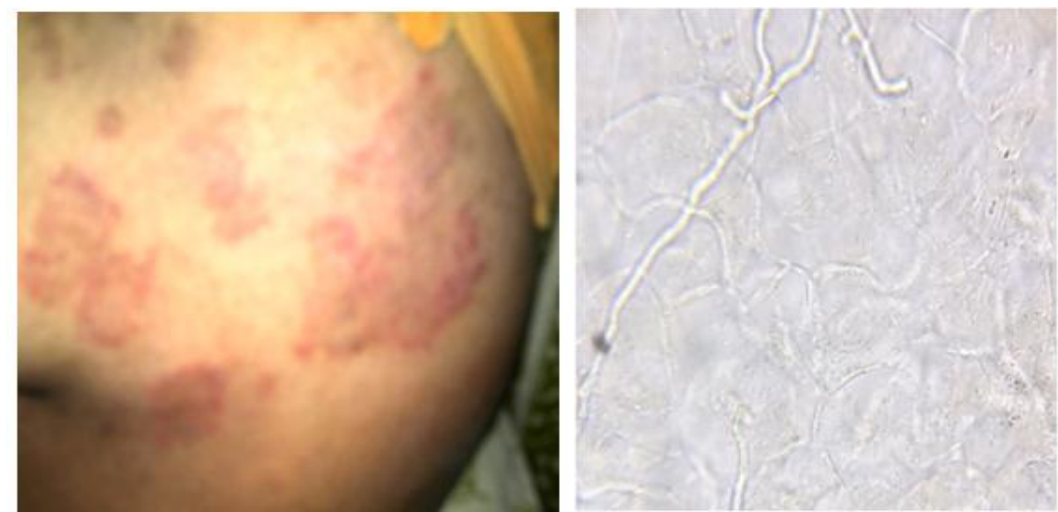

Fig 1\&2 - Case no. 13; Tinea corporis $\quad$ KOH Mount, 40X showing fungal hyphae
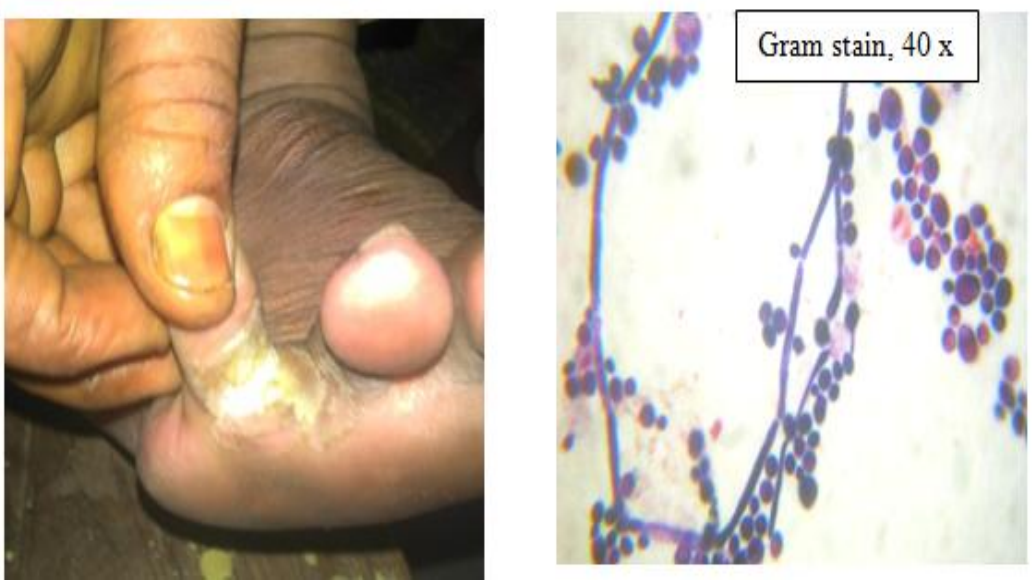

Fig 3\&4, Case no.122 Candidial intertrigo Pseudo hyphae \& budding forms
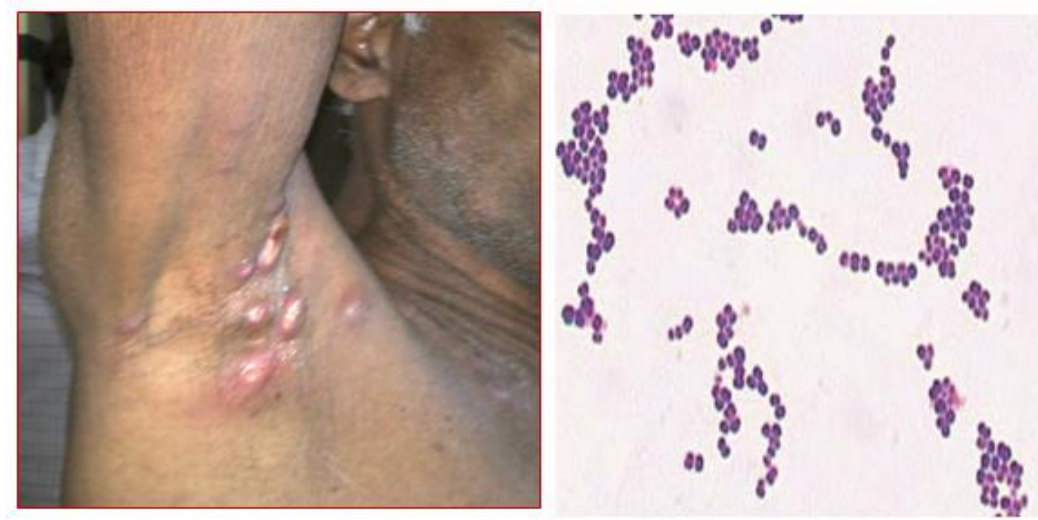

Fig 5\&6 - Case no.40 - Furunculosis Gram stain, 40X showing Staphylococci
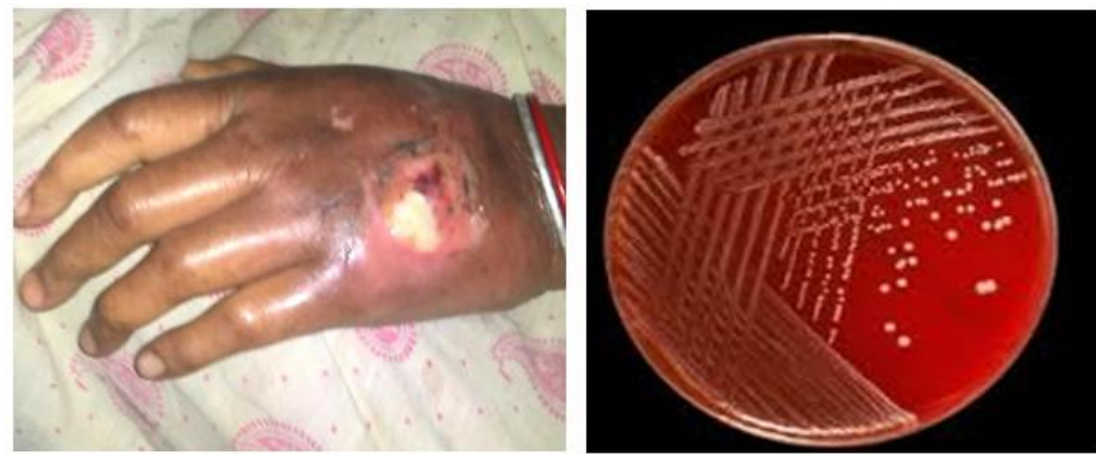

Fig $7 \& 8$ - Case no.36, Diabetic ulcer Staphylococci aureus on blood agar 
Table 1: Gender distribution of cases Total number of cases $(n)=160$

\begin{tabular}{|l|r|c|}
\hline \multicolumn{1}{|c|}{ Gender } & No. of patients & Percentage (\%) \\
\hline Males & 91 & 57 \\
\hline Females & 69 & 43 \\
\hline Total & 160 & 100 \\
\hline
\end{tabular}

Table 2: Classification based on number of mucocutaneous lesions per patient.

Total number of cases $(\mathrm{n})=160$

\begin{tabular}{|c|l|c|c|}
\hline S.No. & \multicolumn{1}{|c|}{ No. of lesions } & No. of Cases & Percentage (\%) \\
\hline 1 & Single lesion & 114 & 72 \\
\hline 2 & Two lesions & 38 & 23 \\
\hline 3 & Three or more lesions & 8 & 5 \\
\hline & Total & 160 & 100 \\
\hline
\end{tabular}

Table No 3: Classification of lesions according to duration of diabetes.

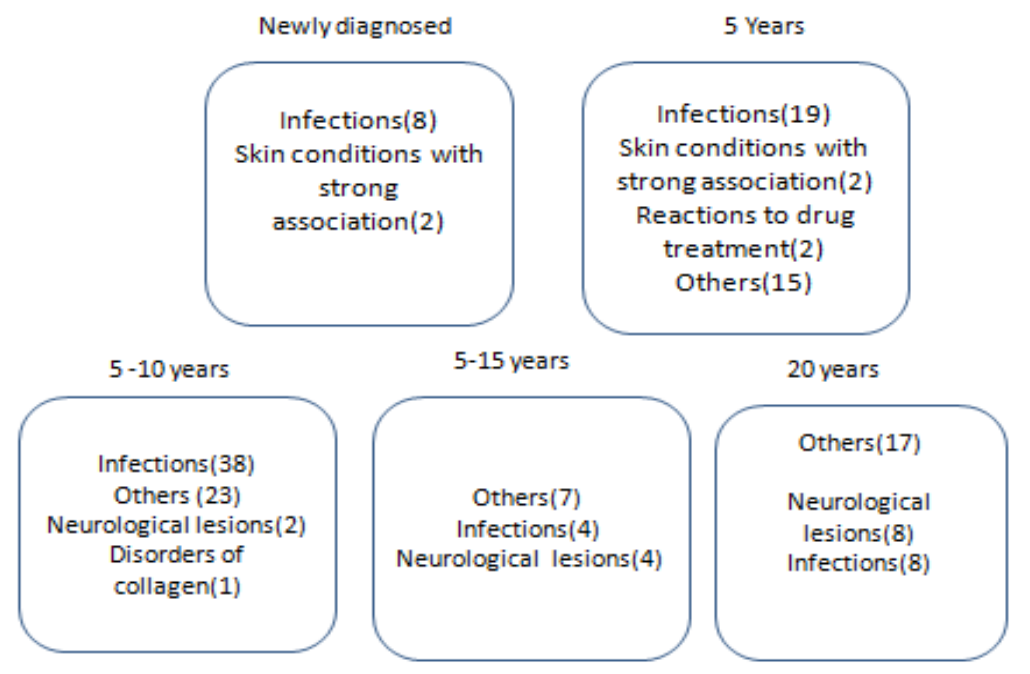

Table 4. Comparision of present study with previous studies:-

\begin{tabular}{|c|c|c|c|c|c|}
\hline Parameters & $\begin{array}{l}\text { Mahajan S } \\
\text { et. al., } \\
\text { NewDelhi }\end{array}$ & $\begin{array}{l}\text { Abhishek } \\
\text { goyal et.al ., } \\
\text { Western } \\
\text { himalayas }\end{array}$ & $\begin{array}{l}\text { Roshini } \\
\text { vahora } \\
\text { et. .al ., } \\
\text { Gujarat } \\
\text { h }\end{array}$ & $\begin{array}{l}\text { N T Foss et } \\
\text {.al., } \\
\text { Brasil. }\end{array}$ & $\begin{array}{l}\text { Present study } \\
\text { AP. }\end{array}$ \\
\hline M:F Ratio & 1: 1.38 & 1.7: 1 & 1.3: 1 & ------ & 1.15:1 \\
\hline Peak Age group & $40-50 y$ & $55-60 y$ & $>40 y$ & -------- & $50-60 y$ \\
\hline $\begin{array}{l}\text { Commonest } \\
\text { skin lesions }\end{array}$ & $\begin{array}{l}\text { Infections } \\
(54 \%)\end{array}$ & 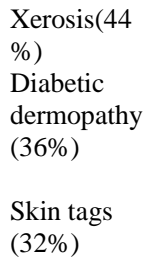 & $\begin{array}{l}\text { Infection } \\
\text { s } \\
(39 \%) \\
\text { Acanthos } \\
\text { is } \\
\text { nigricans } \\
(15 \%)\end{array}$ & $\begin{array}{l}\text { Infections } \\
(82 \%) \\
\text { Actinic } \\
\text { degeneration } \\
(6 \%)\end{array}$ & $\begin{array}{l}\text { Cutaneous } \\
\text { infection(48) } \\
\text { Xerosis }(28 \%) \\
\text { Others }(25 \%) \\
\text { Diabetic } \\
\text { ulcers }(4 \%)\end{array}$ \\
\hline $\begin{array}{l}\text { Patients with } \\
\text { more than one } \\
\text { lesion }\end{array}$ & - & $80 \%$ & $44 \%$ & - & $28 \%$ \\
\hline
\end{tabular}

Graph 1 : Age group distribution of patients Total number of patients (n) $=160$ 


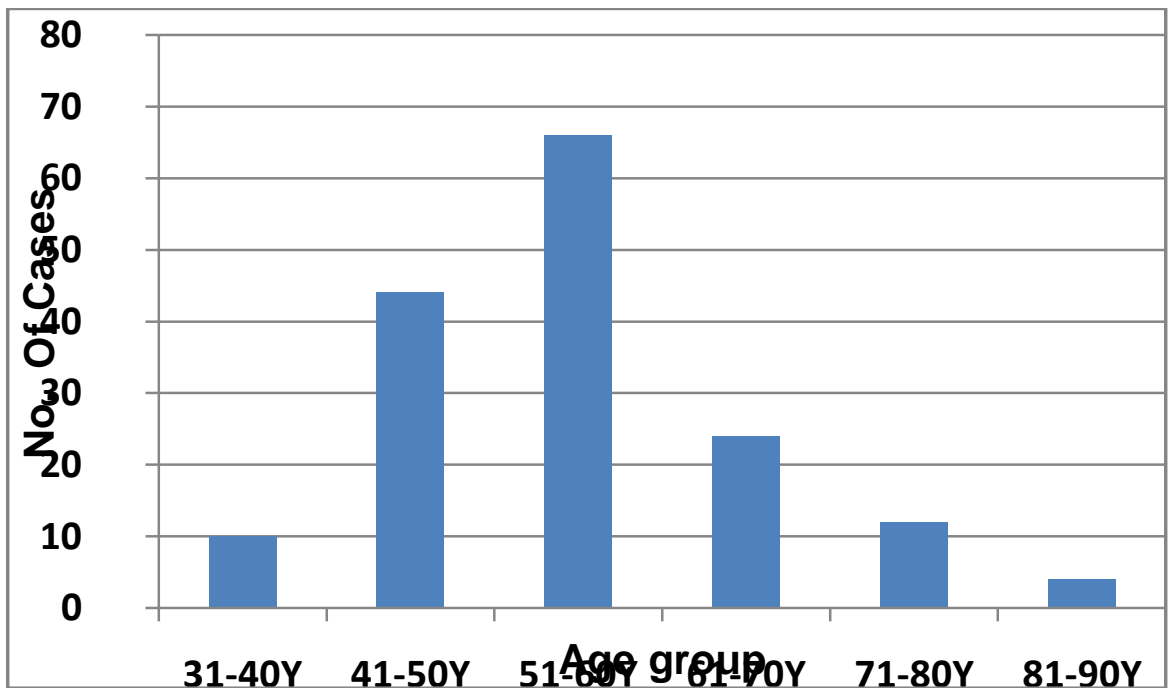

Graph 2: Classification of muco cutaneous lesions

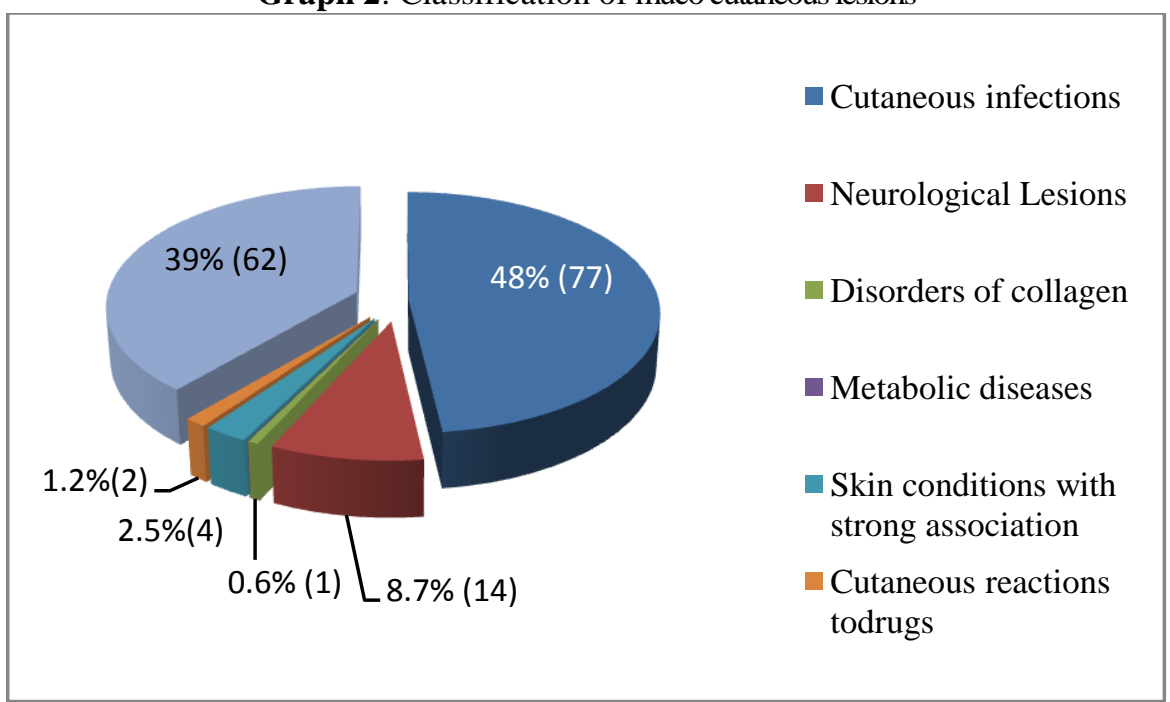

Graph 3: Classification of lesions related to infections. Total no. of cases $(n)=77$

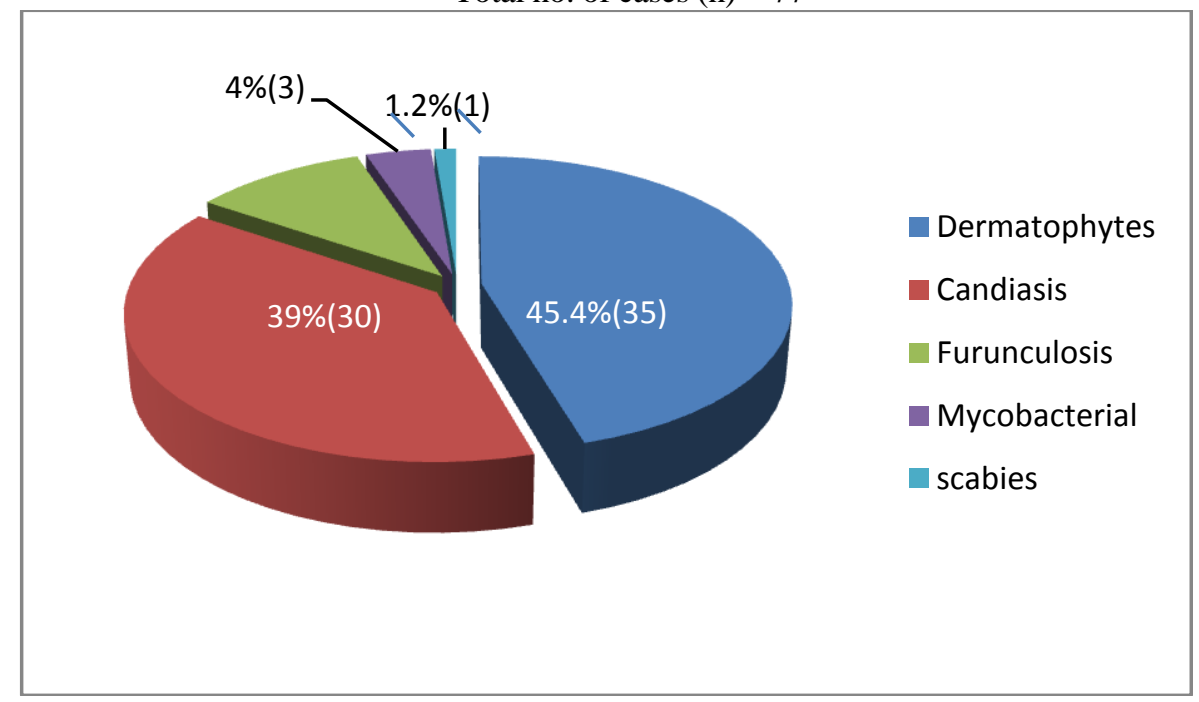

